\title{
DESARROLLO URBANO, COALICIONES DE PODER Y PARTICIPACIÓN CIUDADANA EN BARCELONA: UNA NARRATIVA DESDE LA GEOGRAFÍA CRÍTICA ${ }^{1}$
}

\author{
Antònia Casellas \\ Departamento de Geografía. Universitat Autònoma de Barcelona \\ antonia.casellas@uab.cat
}

\section{RESUMEN}

Con un enfoque desde la geografía crítica, el artículo aborta la evolución de la gobernanza y las estrategias de desarrollo urbano y económico en Barcelona. Se analiza la reorientación paulatina de la política local, desde un modelo de gestión y redistribución social iniciada en los años ochenta, hacia una política dominada por la promoción de imagen y las plusvalías del suelo. Se identifica el punto de inflexión, las características de los agentes involucrados y las dinámicas en la toma de decisiones, abordando los cambios de narrativa promocional y planificación a partir del 2010, desde la escala de ciudad a la escala metropolitana. El artículo concluye con una reflexión sobre la necesidad de una gobernanza local más inclusiva.

Palabras clave: planificación urbana, desarrollo económico, gobernanza, geografía crítica, Barcelona.

\section{ABSTRACT}

From a critical geography approach, the article addresses the debate on the characteristics of governance and urban and economic development strategies in Barcelona. It analyzes the gradual reorientation of local policies, from a management and social redistribution approach that began in the eighties, to a policy dominated by image promotion and capital gains from

Fecha de recepción: noviembre 2013.

Fecha de aceptación: octubre 2014.

1 Esta investigación se ha realizado con el apoyo de los proyectos CSO 2012-31979 y CSO 2013-46863-C3-2-R. La autora desea agradecer los comentarios de un revisor los cuales han contribuido significativamente a la mejora del artículo. 
land development. It identifies the turning point in this process, the characteristics of the agents involved and their decision-making dynamics, addressing the changes in marketing and planning narrative since 2010, from the urban to the metropolitan scale. The article concludes with a statement on the need for more inclusive local governance.

Keywords: urban planning, economic development, governance, critical geography, Barcelona.

\section{INTRODUCCIÓN}

Si bien las ciudades han estado el centro de la civilización, tal y como señala Grange (1999), desde la década de 1990 éstas han aumentado su protagonismo y se han convertido en actores cruciales, tanto de procesos económicos, como sociales y medioambientales. Las ciudades son no sólo los principales centros de producción y consumo (Florida, 2004 y Florida et. al., 2007; Gilbert and Gugler, 1992; Hall, 1998; Sassen, 1991; Scott, 2008), sino que a su vez se han convertido en áreas de creación de emisiones contaminantes directamente vinculadas a procesos productivos y hábitos de consumo (Satterthwaite, 2009). La actual crisis económica añade otra nueva dimensión a esta problemática. La crisis financiera iniciada en el año 2007 se transforma en una crisis fiscal con una fuerte dimensión urbana a finales de la primera década de siglo (Harvey, 2011). Los recortes en inversiones públicas en numerosas ciudades implican un reto importante a corto y medio plazo, que obliga a las ciudades a repensar estrategias, tanto respecto a sus políticas públicas como a sus líneas de desarro1lo. La morfología urbana se sigue revelando como especialmente importante, en cuanto se acrecienta la necesidad de implementar un modelo de ciudad con una buena provisión y asignación de usos mixtos y actividades. Pero, de forma muy especial, ante la necesidad de estrategias novedosas, se hace imperante el estudio de las características de los agentes y los procesos de decisiones en la gobernabilidad local.

Con la voluntad de revaluar el proceso evolutivo en políticas locales y abrir el debate a nuevas posibilidades de desarrollo y gobernabilidad urbana, en los siguientes apartados se explora la dialéctica existente entre restructuración urbana, crecimiento económico y gobernanza, estudiando el caso de Barcelona. El análisis se realiza aplicando el modelo analítico aportado por David Harvey (1989, 2010 y 2011) referente al progresivo cambio de role que ha sufrido el sector público. Se analiza por qué y cómo Barcelona, a pesar de haber presentado una imagen de política local progresista, no ha escapado a una transformación paulatina de su política urbana, a través de la cual el sector público ha reorientado su enfoque de gestión y redistribución social iniciada en los años ochenta, hacia una política urbana dominada por intereses de promoción económica y plusvalías del suelo ligadas a la burbuja inmobiliaria española de finales de la década de los noventa y la primera década del siglo veintiuno. Se identifica el punto de inflexión en este proceso y se estudia la estrecha relación que la ciudad ha mantenido entre la estrategia de urbanización del suelo y la promoción económica. La investigación aborda en su parte final los motivos de cambio de narrativa en política pública, desde la escala de ciudad a la escala metropolitana, y apunta a la necesidad de una nueva reorientación en políticas públicas. 
El caso de Barcelona es emblemático en la medida en que el proceso de transformación de la ciudad, tanto urbanístico como económico, ha sido extraordinario, y en muchos casos se ha estudiado como modélico (González, 2010; Marshall 2000 y 2004). A su vez, el análisis de la evolución de las políticas urbanas y económicas en Barcelona aporta un gran potencial analítico, ya que el inicio del periodo democrático y sus políticas urbanas se producen de forma paralela a la transformación del capitalismo a partir de la crisis del petróleo de los años setenta. En el nuevo escenario surgido a partir del 2007, el análisis de la capacidad de respuesta de la ciudad de Barcelona permite estudiar, contextualizado dentro de su proceso evolutivo, potencialidades y debilidades de las políticas urbanas en un periodo de cambio de paradigma socio-económico.

El análisis se elabora a partir de la recopilación y análisis de información aportada por fuentes primarias y secundarias. Las fuentes primarias incluyen la observación no participante y entrevistas semi-estructuradas. Los argumentos que diferentes colectivos han manifestado acerca del proceso de restructuración urbana y metropolitana se reconstruye a través de nueve entrevistas semi-estructuradas realizadas a agentes económicos y sociales. Cuando se ha considerado necesario, y con el fin de garantizar el anonimato, se identifica a los informantes con un número. Así mismo, se abordan tres casos de estudio relacionados con la transformación urbanística de la ciudad - los Juegos Olímpicos del 92, el Fórum de las Culturas 2004, y el Proyecto 22@ Barcelona- los cuales permiten contextualizar el debate. La fuente documental incluye el análisis de abundante material que permite el estudio de estrategias de desarrollo económico y urbano. Respecto a la estructura del artículo, después de esbozar el marco conceptual del análisis, se analiza la evolución y configuración del modelo de gobernanza local en Barcelona con especial énfasis en el papel de agentes políticos y económicos, la cooperación público-privada y las principales líneas de desarrollo económico. A continuación se evalúan los proyectos iniciados a partir del 2000, su vinculación al desarrollo del suelo y la nueva narrativa a escala metropolitana surgida a principios de la década del 2010. El artículo concluye con unas reflexiones sobre la dialéctica generada entre competitividad y gobernanza.

\section{EL DEBATE EN TORNO A LA GOBERNANZA URBANA Y SU PROCESO DE RECONFIGURACIÓN}

Una de las consecuencias de la restructuración del capitalismo a partir de la crisis del petróleo de los años setenta ha sido la progresiva pérdida de peso de los estados como instituciones reguladoras tradicionales, con una evolución hacia una posición de coordinación de intereses múltiples entre actores económicos y sociales (Caravaca y González, 2009; Jessop, 2002). En este proceso, aunque las grandes urbes y áreas metropolitanas se siguen considerando centros neurálgicos de actividad económica (Florida et al., 2007), se observa un mayor rol ganado por las ciudades de dimensiones medias, las cuales presentan capacidades de resiliencia resultantes de una nueva reformulación de agentes y estrategias de innovación (Méndez, 2013). Esta nueva complejidad hace necesario remarcar la presencia de crecientes conflictos de redistribución entre diferentes escalas de gobernabilidad (Milio, 2013), obligando a repensar mecanismos de representatividad social y gobernanza urbana (Gerometta et al, 2005; Farinós, 2008, Le Galès, 2002). 
La tensión entre niveles de gobernabilidad se ejemplifica con la presencia de estructuras políticas de niveles superiores al estado, como la Unión Europea; o comerciales, como NAFTA (Hooghe y Marks, 2003) y las reivindicaciones de escalas inferiores a las de los estados tradicionales. A escala inferior se han incrementado las reivindicaciones políticas regionales y locales, y se ha abogado por el principio de subsidiaridad, reivindicado no solo desde las regiones o municipios, sino articulado desde macro-estructuras como la Unión Europea, quien desde el tratado de Maastricht de 1992 y la reformulación del tratado de Lisboa en 2009, interpreta el principio de subsidiaridad como base de la democracia participativa. Vinculado a esta nueva reformulación de poder y atributos, la restructuración del modelo capitalista ha comportado asimismo un proceso paulatino de globalización económica y un cambio en la relación entre el sector público y la ciudadanía, en la medida en que se han ido imponiendo paulatinamente estrategias de autoorganización y autoregulación, tanto a nivel institucional como personal (Jessop, 2002, Hacker, 2006, Hajer, 2003, Moulaert et al, 2007; Swyngedouw, 2005). Uno de los elementos fundamentales en este cambio a escala local ha sido la aparición de estilos de gobierno en los que los límites entre los sectores público y privado se han difuminado, a la vez que se ha hecho patente una paulatina transformación del estado del bienestar hacia un estado de carácter más emprendedor, el cual ha implicado la priorización de estrategias de desarrollo de carácter económico en detrimento de políticas de carácter social (Stoker, 1998, Harvey, 2010).

En este contexto, tanto a nivel académico como dentro de la gestión pública se ha generalizado el concepto de gobernanza, como propuesta alternativa al papel tradicional de gobierno. Healey (2007) argumenta que la gobernanza local implica la articulación de las normas de comportamiento con respecto a los asuntos colectivos de una comunidad política y de los principios de asignación de recursos entre los miembros de la comunidad. Pierre y Peters (2000) argumentan que la popularidad del concepto de gobernanza deriva de que, a diferencia del término más estrecho de gobernabilidad, el primero posee una capacidad para cubrir toda la gama de instituciones y relaciones involucradas en el proceso de gobernar. El concepto implica, en cualquier caso, la necesidad de que las políticas se deban adaptar a las peculiaridades de los territorios y sus agentes (Farinós, 2008).

Dentro de esta dinámica, en un proceso que se inicia a mediados de los años ochenta, el sector público en los países occidentales redefine paulatinamente su rol y cambia su papel de proveedor de mecanismos de planificación y prestación de servicios sociales, a emprendedor e innovador económico. En Baltimore, uno de los primeros casos emblemáticos, Harvey (1989) identifica tres factores que le permiten definir el creciente carácter empresarial del sector público y sus políticas urbanas. En primer lugar destaca el incipiente papel de la colaboración público-privada en el centro de las políticas públicas, las cuales por primera vez combinan y comparten atributos y recursos propios de la administración pública y los sectores privados, creando gobiernos a la sombra. Este punto de inflexión en la colaboración público-privada se genera en Baltimore como resultado del referéndum celebrado en 1978 que aprobó, por un corto margen, la utilización de propiedad pública para la reconstrucción de su frente marítimo liderada por el sector privado. En segundo lugar, se identifica el carácter especulativo de la colaboración público-privada. En la medida en que la actividad no está orientada a la creación de infraestructura o actividad económica vinculada a la comunidad existente, el riesgo se transfiere básicamente al sector público. Esta es una faceta que dife- 
rencia la actitud del sector privado de fases anteriores, en las que no se mostraba tanta aversión al riesgo, ya que éste se incorporaba como parte intrínseca de la actividad empresarial. Por último, en el caso de Baltimore el nuevo carácter empresarial de las políticas públicas se caracteriza por perder interés en proyectos orientados a la mejora de calidad de vida o creación de empleo, para concentrarse en la creación de imagen que trasciende el espacio concreto en el que se sitúan.

En el caso de Barcelona es también posible identificar este proceso dentro de su evolución temporal. Si bien a finales de los años setenta la ciudad se enfrentaba a numerosas deficiencias en infraestructura y servicios, sin haber sufrido grandes procesos de renovación urbana que afectaran su patrimonio urbanístico, como sucedió en numerosas ciudades anglosajonas, la ciudad contó con herramientas de planificación y de gestión necesarias para afrontar la mejora urbanística (Calavita y Ferrer, 2000; Casellas, 2003). Sin embargo, a partir de la nominación en 1986 de Barcelona como sede olímpica para los juegos de verano de 1992, el sector público inició un proceso de transformación urbana a gran escala. Gracias al éxito organizativo y promocional de los juegos, y la consecuente proyección mediática de la ciudad, la transformación urbanística de Barcelona generó gran interés a nivel internacional². Respondiendo a este interés hacia Barcelona como ejemplo de buenas prácticas, la promoción internacional se ejemplifica en el libro editado por Jordi Borja (1996) y encargado por el Banco Mundial y el Programa de Gestión Urbana de Naciones Unidas que, bajo el título «Barcelona, un modelo de transformación urbana», inaugura el debate sobre la creación de un determinado modelo de urbanismo en Barcelona ${ }^{3}$.

En la transformación urbana y económica de Barcelona, el estudio de los agentes y políticas públicas permite identificar un proceso evolutivo que afecta tanto las características de

2 En 1993, la creación del Centro Iberoamericano de Desarrollo Estratégico Urbano (CIDEU) generó una red urbana en la que numerosas ciudades tuvieron la oportunidad de acceder y explorar modelos de desarrollo urbano catalogados como de mejores prácticas. En la década siguiente, agentes directamente involucrados mayoritariamente desde el sector público en la transformación urbana de la ciudad promocionaron el éxito de la renovación de Barcelona, el cual fue ampliamente reconocido en el ámbito académico e institucional internacional. Con este fin, desde los años noventa, académicos, políticos y especialistas de administraciones públicas han visitado Barcelona con el objetivo de aprender de la ciudad y aplicar modelos parecidos de ordenación territorial y dinamización económica (González, 2010). Con el tiempo, como señala Borja (2007), bajo el concepto de «modelo Barcelona», la ciudad fue progresivamente identificada como un ejemplo de desarrollo urbano, tanto por agentes internos al proceso, como también por la necesidad de otras ciudades de encontrar experiencias que sirvieran de ejemplo y de legitimación a sus procesos de renovación y proyectos urbanos.

3 En el curso 1997-1998, en las facultades de economía y arquitectura de la Università degli Studi Roma Tre, se celebraron tres seminarios bajo la dirección de Pasqual Maragall. La idea partía de la necesidad de establecer un espacio de debate y reflexión que se materializó en tres seminarios. Tanto en el seminario en Italia como en publicaciones de este periodo, se observa claramente el esfuerzo, por parte de los responsables públicos de la transformación de la ciudad, de difundir la experiencia de Barcelona como ejemplo de planificación urbana a seguir. Los aspectos en política urbana que se destacan incluyen: 1) la renovación urbanística de los espacios degradados del centro histórico y la periferia de la ciudad, liderada por el arquitecto Oriol Bohigas; 2) el posicionamiento internacional de la ciudad y la creciente importancia de la nueva tecnología para el desarrollo económico local; 3) la implicación de los ciudadanos en la transformación de la ciudad a partir de lo que se identifica como participación ciudadana, 4) la utilización de los grandes acontecimientos y la cultura como estrategias de transformación; 5) la integración entre lo público y lo privado; 6) la incorporación de la planificación estratégica; 7) la cooperación ciudad-universidad-empresa; y por último, 8) la importancia de la legislación como herramienta de gobernabilidad (Acebillo 1999; Bohigas, 1999 y 2004; Busquets, 2004; Esteban, 1999, 2003; Maragall, 1999, 2008; Santacana, 1999). 
los agentes como las relaciones entre éstos, y que indica un creciente dominio de los intereses económicos sobre las políticas de equidad social (Capel, 2005 y 2007; Casellas, 2006; Blanco, 2009; Montaner, 2004). En este proceso, el sector público, y más concretamente el ayuntamiento mantiene su papel de liderazgo, tanto en el ámbito urbanístico (Mauri y Uría, 1998; Moix, 1994; Monclús, 2003; McNeill, 1999 y 2003), como en el de promoción económica (Casellas y Pallares, 2009; Pareja-Eastaway y Piqué, 2011). Sin embargo, la evolución en la gobernanza local involucra paulatinamente agentes políticos y técnicos hacia un difuminado pero altamente eficiente proceso de cooperación público-privada que se consolida a partir de los años noventa del pasado siglo. El análisis de esta evolución se aborda en el siguiente apartado.

\section{LA EVOLUCIÓN EN EL ROL DEL SECTOR PÚBLICO: DE GESTOR URBANO A EMPRENDE- DOR ECONÓMICO}

\section{III.1. La etapa de gestión. De la recuperación de los barrios a la reurbanización de la ciudad}

El liderazgo del sector público en Barcelona se inicia con las primeras elecciones locales democráticas celebradas en 1979. Después del largo periodo de dictadura franquista, con una administración local enfrentada a un gran déficit de infraestructuras y servicios y con escasos recursos económicos, la reforma y racionalización de la administración se presenta como prioritaria. La descentralización de funciones del gobierno local a escala de barrio, y las pequeñas actuaciones urbanísticas de bajo presupuesto en los espacios públicos más degradados son las actuaciones más destacadas de este periodo. Como identifica el Informante 4 (2012) y recogen diferentes fuentes bibliográficas (Bohigas, 1985, 1999; Moix, 1994; Montaner, 2003 y 2004), las reivindicaciones de los movimientos vecinales influyen en las prioridades de la política local, a la vez que arquitectos y académicos que han jugado un papel relevante en los movimientos sociales organizados a través de protestas vecinales reivindicativas durante la última etapa del periodo franquista se identifican como individuos clave en la administración local, determinando la visión y ejecución de proyectos urbanísticos, con un énfasis en la mejora de la calidad de vida de los ciudadanos y la provisión de servicios.

Desde el punto de vista urbanístico, en este periodo se da prioridad a la recuperación del centro de la ciudad y la revalorización de la periferia, llevada a cabo bajo el criterio del método proyectual. La idea de proyecto urbano como método se basa en ejecutar mejoras urbanísticas gracias a la concreción. De esta forma se realizan proyectos que se consideran fiables y programados operativamente. La filosofía proyectual parte del estudio de un barrio o un sector de éste, lo cual ayuda a determinar las características funcionales y simbólicas del espacio, y establecer la predisposición morfológica y funcional, para concretar las actuaciones a través del proyecto (Bohigas, 1999). En este proceso inicial de restructuración urbana, el espacio público se entiende como el punto de partida de las intervenciones públicas. Por oposición a un urbanismo descontextualizado y segregado, se reivindica el concepto de calle, plaza, espacio urbano y tipología arquitectónica propios de la ciudad tradicional (Esteban, 2003). Proyectos puntuales en barrios del centro de la ciudad, como la Plaça de la Mercè en el Barrio Gótico, y en la periferia, como la Via Júlia, permiten recomponer y dignificar espa- 
cios urbanos degradados, y simbolizan la recuperación de los espacios públicos que posteriormente merecerán el reconocimiento internacional de la Universidad de Harvard en 1990, y del Instituto Real de Arquitectos Británicos en 1999. Con relación a los servicios sociales, el ayuntamiento lleva a cabo la creación de nuevos servicios para poblaciones específicas, con especial énfasis en la juventud y la tercera edad ${ }^{4}$. Esta primera etapa, que en la terminología de Harvey, podría definirse de gestora, abarca desde 1979 a 1986, año en el que se produce la nominación de Barcelona como sede de los juegos olímpicos de verano de 1992.

\section{III.2. Punto de inflexión y proceso de aprendizaje en la cooperación público-privada}

Harvey identifica el referéndum de 1978 como punto de inflexión en la política de Baltimore hacia el modelo neoliberal definido anteriormente. En el caso de Barcelona, el punto de inflexión se puede ubicar en la creación de la primera agencia público-privada para la construcción de la infraestructura olímpica en 1988. Como apunta el Informante 6 (2012), liderado por partidos de izquierdas en posiciones de poder después de años en la oposición clandestina al régimen franquista, a mediados de los años ochenta, la administración local se considera representativa de las clases obreras y los movimientos ciudadanos. Pero, a su vez es consciente de que no cuenta con el respaldo empresarial, el cual mantiene reticencias hacia unos grupos políticos que considera ideológicamente extremos (Raventós 1998 y 2000). En este contexto, los juegos se presentan como una gran oportunidad de ejecutar en un plazo de tiempo corto una transformación urbana de ciudad a gran escala, pero para ello el sector público necesita la inversión privada. Esta necesidad de movilizar recursos y de asegurar la cooperación del sector privado crea las condiciones para una tentativa inicial de cooperación entre el sector público y el privado. La primera agencia mixta entre capital público-privado en Barcelona, NISA, surge en 1988 del compromiso entre el ayuntamiento e inversores privados para financiar y ejecutar la edificación de la Villa Olímpica como complejo de viviendas que, aunque inicialmente pensados como vivienda social, posteriormente se venderán a precios de mercado a inversores privados y familias ${ }^{5}$. A partir de esta primera iniciativa, el proceso de aprendizaje en la cooperación público-privada se expande hacia la transformación urbanística del centro histórico. Simultáneamente numerosas fundaciones privadas registradas como no gubernamentales se organizan para actuar como asesoras y colaborar en fomentar nuevos sectores económicos, en colaboración con la administración pública (Informante 1, 2007).

En este periodo, la planificación estratégica de Barcelona se constituye una de las herramientas urbanísticas clave, la cual posteriormente suscitará un gran interés tanto a nivel internacional como nacional. En el contexto del primer plan estratégico, como

4 Los nuevos servicios incluyen la creación de centros culturales y la construcción de instalaciones deportivas, así como centros residenciales. Los centros cívicos a escala de barrio son planificados en colaboración con las organizaciones cívicas. Respondiendo a las necesidades sociales, los servicios ofrecidos por los centros incluyen cursos de alfabetización, servicios a domicilio para personas mayores y eventos culturales, entre otros. Estos servicios a menudo son financiados por el municipio y gestionados a través de organizaciones de la comunidad (Borja, 1996).

5 Un primer resultado de la dinámica de esta cooperación se materializa en las viviendas olímpicas que, inicialmente pensadas como vivienda social y llamadas Nova Icaria en homenaje al socialista utópico Etienne Cabet (Balcells, 1986), bajo las exigencias del capital privado y las constructoras se venderán a precio de mercado después de la celebración de los juegos. 
reconoce Raventós (1998 y 2000), desde la administración local se había generado una voluntad de evitar la desaceleración de la economía como consecuencia de la resaca postolímpica. Para ello, uno de los objetivo es tener una visión compartida entre un amplio abanico de agentes económicos y sociales que permita capitalizar la dinámica generada con la creación de nueva infraestructura y la visibilidad internacional conseguida con los juegos. En este sentido, a partir de esta primera iniciativa, la planificación estratégica se seguirá manteniendo como herramienta de consenso público-privado, con la aprobación de planes estratégicos tanto de carácter territorial como sectorial. Entre otros, destacan el segundo y el tercer plan estratégico de Barcelona, plan estratégico del sector cultural (1999), deportes (2002), turismo (2008) y el plan estratégico metropolitano de Barcelona, Visión 2020, aprobado el 2010. El diseño de esta planificación estratégica y sectorial, aunque incluye sectores amplios de la sociedad civil, condiciona por su carácter técnico la efectividad de la participación ciudadana (Informante 7, 2012; Informante 9, 2013). Por ello se puede argumentar que la planificación estratégica ha funcionado como mecanismo por parte de la administración local de inclusión en el proceso de decisión política de un amplio espectro de agentes económicos y sociales, pero dando prioridad a la creación de consenso y a la presencia de actores que aporten recursos, sean de carácter económico o técnico en detrimento de la participación ciudadana.

Esta dinámica de decisión genera que el crecimiento económico y la restructuración urbanística en Barcelona paulatinamente se vea condicionada por la formación de preferencias de los agentes con recursos (Casellas, 2005). Las preferencias en políticas públicas se van configurando a través del proceso de colaboración público-privado y las tendencias de globalización de la economía, que prioriza las políticas locales de desarrollo económico (Harvey 1989 y 2010). Desde esta perspectiva se observa que en Barcelona la capacidad de ejecutar proyectos es el resultado de un reconocimiento de interdependencia y respeto mutuo entre la administración local y los sectores privados que aportan capital inversor y experiencia ejecutora (Andreu, 2011; Casellas, 2007).

En el periodo postolímpico la dinámica de cooperación público-privada se consolida y las tensiones entre redistribución social y restructuración urbanística se intensifican (Sabaté Bel y Tironi Rodó, 2008). En la prolongación de la Avenida Diagonal, proyecto relevante para la futura expansión del frente marítimo de la ciudad y la remodelación económica y física del tradicional barrio industrial y obrero del Poblenou, el equipo de arquitectos encargados inicialmente del proyecto renuncia como protesta a la desaparición de vivienda social y el nuevo modelo de gestión adoptado para ejecutar el proyecto. En la dialéctica entre el sector público y privado, la aversión del sector privado al riesgo se hace patente en la medida en que «los agentes privados asumen la transformación del área a cambio de rebajar demandas sociales y se atribuyen aquellas unidades que ofrecen menores dificultades de gestión y costes. Las áreas más consolidadas, las más resistentes quedan para ser gestionadas por el ayuntamiento» (Sabaté Bel y Tironi Rodó, 2008: 248).

El carácter de la inversión privada muestra en el período postolímpico, tal y como apuntaba Harvey en el caso de Baltimore, una creciente reticencia al riesgo y un carácter especulativo que, en el contexto de Barcelona — dentro de la dinámica económica y las regulaciones del estado español en materia de suelo - se orienta hacia la urbanización del suelo, un proceso incentivado por el beneficio que ofrece el diferencial de rentas, teorizado extensamente 
por Smith (1987), y fomentado por la burbuja inmobiliaria del país a partir de 1997, con un marco legal a escala nacional caracterizado por la desregularización y la deslegitimación de la planificación (Burriel, 2008 y 2011).

\section{III.3. La estrategia de crecimiento económico vinculada a la urbanización del suelo y la creación de imagen}

A finales de los años noventa, el éxito promocional y urbanístico que acompaña a los juegos olímpicos y la necesidad de buscar nuevas estrategias de crecimiento económico, dentro de la dinámica de cooperación público-privada establecida, se concreta en la utilización de dos estrategias complementarias. Por un lado, se actúa a través de la restructuración urbanística de grandes dimensiones, capitalizando en las plusvalías inmobiliarias como atractivo inversor para el sector privado; y por otro, se acentúa la creación de imagen, en terminología de Harvey (1989), la «creación de espacio» a través de la marca Barcelona, que tiene como objetivo promocionar la capacidad turística, residencial e inversora de la ciudad (García, 2013).

En esta línea se puede interpretar la celebración del macro-evento Fórum de las Culturas 2004 que, bajo el pretexto de acoger un evento cultural de visibilidad internacional durante varios meses, permite la transformación urbanística de unas 240 hectáreas de frente marítimo, al lado del río Besòs. El evento tiene lugar en un espacio históricamente degradado de la ciudad que combina la presencia de infraestructura contaminante - planta depuradora, central térmica, depósito de residuos - junto a un barrio marginal. El evento funcionó como pretexto para movilizar recursos públicos y privados y ayudó a la transformación de una zona con una problemática de contaminación ambiental grave. La idea inicial, como apunta Borja (2005), parecía bien intencionada ya que permitía modernizar las instalaciones y dignificar una zona problemática. El resultado final de la iniciativa del Fórum, sin embargo, fue ampliamente cuestionado, tanto desde un punto de vista organizativo como urbanístico (Andreu Acebal, 2008; Delgado, 2004 y 2007).

A nivel organizativo se evidenció la contradicción entre la propuesta temática del evento y su narrativa promocional. El Fórum giraba alrededor de temas de multiculturalidad, sostenibilidad y paz, pero se hizo manifiesta la falta de voluntad de inclusión de la ciudadanía por parte del ayuntamiento (Andreu Acebal, 2008). En el año 2001, el movimiento vecinal organizado a través de la Federación de Asociaciones de Vecinos y Vecinas de Barcelona (FAVB) propuso al ayuntamiento ideas específicas que, partiendo de un bottom-up participativo, ayudaba a articular el proceso de participación de amplias capas de la población. Sin embargo, el ayuntamiento optó primero por el silencio administrativo y después por la negativa acompañada de presiones políticas. El Fórum se celebró finalmente con el patrocinio de empresas multinacionales, inversión pública y abundante presencia de ponentes de prestigio internacional, junta al descontento de amplias capas de la ciudadanía (Delgado, 2004). Con respecto a la calidad de la transformación urbanística de la zona, fueron cuestionados tanto el edificio que debía actuar como creador de imagen promocional diseñado por Herzog y Meuron, como la integración de la reforma global con el espacio colindante (Delgado, 2004 y 2007; Borja, 2005).

Desde la perceptiva de desarrollo del suelo y atracción de capital inversor interesado en plusvalías, se puede argumentar que el evento, sin embargo, funcionó como mecanismo 
para justificar una amplia inversión pública que, iniciada en los años noventa, era condición previa para atraer inversores privados a un proyecto urbanístico adyacente a la zona Fórum. Construido en 1996 por la multinacional americana Hines, el proyecto Diagonal Mar involucra 1.400 viviendas divididas en 5 grandes bloques para un mercado de clase media-alta, 3 hoteles con una capacidad total de 950 habitaciones, 3 bloques de oficinas y un parque público, el cual a pesar de contar con 14 hectáreas, ser el tercer parque de la ciudad y haber sido diseñado por el prestigioso equipo del arquitecto Enric Miralles, inicialmente fue poco frecuentado, dado su diseño interior y escasa conectividad con el tejido urbanístico existente en la zona (Borja, 2005). A este proyecto urbanístico de Hines hay que añadir un colindante centro comercial inaugurado en noviembre del 2001, el mayor de Cataluña hasta aquella fecha, con una superficie alquilable de cerca de $88.000 \mathrm{~m}^{2}$, un hipermercado de $27.000 \mathrm{~m}^{2}$ y un aparcamiento para 5.000 coches. El análisis de las noticas publicadas por la prensa económica acerca de este proyecto comercial permite observar cómo el centro se insirió desde sus inicios en la dinámica financiera global. Hines aceptó la iniciativa del proyecto en 1996 bajo el compromiso de cooperación de un especialista en la gestión de áreas comerciales, el promotor francés Jean Louis Solal, comprando los terrenos a Kepro Internacional por 27 millones de Euros (Apce, 2013). Un año más tarde, Hines adquirió un compromiso de compraventa con Deka, un fondo inmobiliario alemán, quién lo adquirió tres años más tarde, en el 2004, por 240 millones de Euros. Este último a su vez lo vendió en el 2006 al grupo irlandés Quilan Private por 300 millones de Euros (Pérez, 2006). Al estallar la burbuja inmobiliaria irlandesa, Quinlan Private debía 1.500 millones de euros al Anglo Irish Bank. La quiebra de esta entidad financiera convirtió a Nama, el banco malo Irlandés, en propietario de Diagonal Mar. En 2013, Nama empezó a sondear fondos internacionales para vender el activo valorado en 286 millones (El Condifencial, 2013).

Otro de los proyectos que ilustran la estrecha relación existente en la ciudad entre desarrollo económico y urbanización del suelo es el proyecto 22@Barcelona, iniciado en el año 2000. En un espacio de casi 200 hectáreas, el proyecto se presenta como la transformación de un barrio industrial obsoleto en un nuevo centro de actividades vinculadas a la economía del conocimiento. La planificación de usos mixtos del suelo combina actividades productivas, recreativas, vivienda y servicios. El incentivo para la inversión privada procede de la recualificación del suelo y el aumento del índice de edificabilidad. Se incluyen instalaciones para ubicar actividades de economía del conocimiento, definidas de forma amplia. Con el tiempo, la formulación de las estrategias y la narrativa entorno al proyecto evoluciona en función de nuevas alternativas y retos (Casellas y Pallares-Barbera, 2009). Desde una perspectiva productiva, los datos de la sociedad municipal 22 ARROBA BCN, S.A.U., creada en el año 2000 por el Ayuntamiento de Barcelona con la finalidad de gestionar todo el proceso de transformación y entrar en partenariados público-privados, establece que tras un periodo de diez años se han ubicado en la zona un total de 7.064 empresas, con 90.000 trabajadores en plantilla y 4.400 autónomos (22@Barcelona, 2012). Respecto al año 2000, cuando el barrio contaba con 3.437 empresas, el crecimiento empresarial en los diez primeros años se considera altamente exitoso; muy por encima del de la provincia de Barcelona (57,3\%) y el conjunto de Catalunya (60\%) (Pareja-Eastaway y Piqué, 2011). Sin embargo, cabe destacar que del total de estas nuevas empresas, sólo 47,3\% son de nueva creación, ya que el resto es resultado de reubicación de empresas. Algunos de los análisis a 


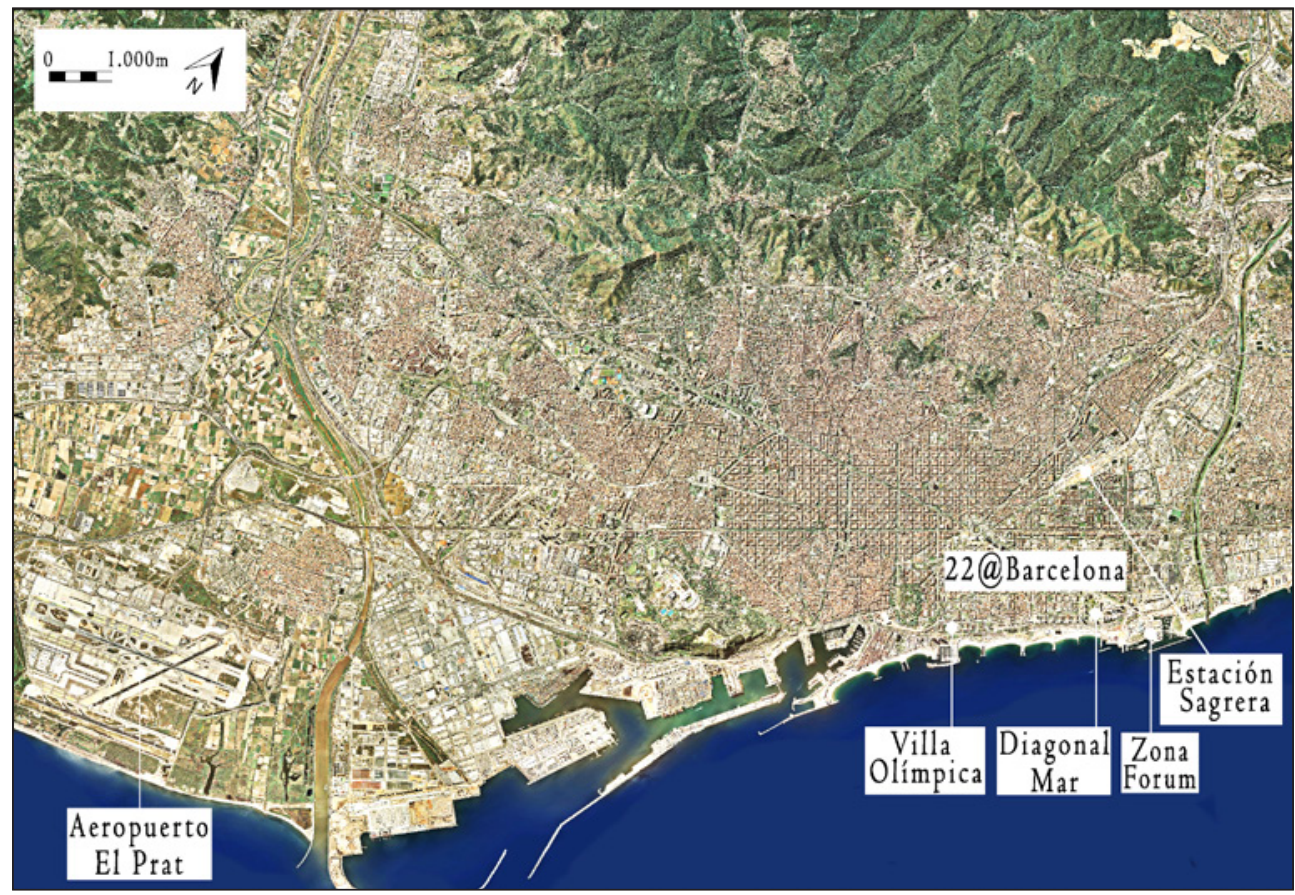

Fuente: elaboración propia a partir de imagen del Institut Cartogràfic de Catalunya.

nivel productivo también apuntan a efectos de gentrificación, tanto de actividad productiva de pequeños talleres y logística, como artísticos, resultado del incremento del precio del suelo (Dot, et al, 2010, Martí-Costa y Pradel Miquel, 2011).

Cabe destacar que, al margen del ámbito productivo, desde el inicio del proyecto 22@ Barcelona un elemento básico en planificación es la combinación de actividades de promoción económica vinculadas a las plusvalías del suelo e inversiones inmobiliarias, las cuales facilitan la atracción de capital privado. En el año 2012, y en plena crisis de la burbuja inmobiliaria española, la web promocional del proyecto insistía en que «es el proyecto de transformación urbanística más importante de la ciudad de Barcelona en los últimos años y uno de los más ambiciosos de Europa de estas características, con un potencial inmobiliario alto y una inversión pública del plan de infraestructuras de 180 millones de euros»(22@ Barcelona, 2012).

Podemos constatar que en el caso de Barcelona, tanto en el proyecto del Forum como en el del22@Barcelona se ha producido una transferencia de riesgo desde el sector privado al sector público, quien ha sido el encargado de crear condiciones atractivas para el capital. Esta misma tendencia se ha manifestado en los procesos de reforma del centro histórico. Desde la sociedad civil y las plataformas ciudadanas esta experiencia urbanística ha recibido amplias críticas y ha propiciado la creación de plataformas cívicas y reivindicativas (Cruz y Martí-Costa, 2010). Entre los aspectos críticos se ha apuntado al cambio de prioridades 
en política urbana que ha menoscabado actuaciones de redistribución social (Borja, 2010; Delgado 2004, 2005 y 2007) y la tematización orientada hacia criterios comerciales basados en valores abstractos ligados a una imagen de marca y la destrucción de patrimonio arquitectónico (Grup Patrimoni Industrial del Fòrum Ribera del Besòs 2005a, 2005b). También se ha cuestionado el proceso de segregación socio-espacial (Alabart Vilà, 2010; Montaner 2004; Tapada-Berteli y Arbaci, 2011) y la marginación de la participación efectiva ciudadana en el proyecto de ciudad (Unió Temporal d'Escribes, 2004).

\section{LA ESCALA METROPOLITANA COMO IMAGEN PROMOCIONAL}

Después de años de reivindicación de la escala metropolitana por parte de sectores económicos y políticos de la ciudad (Borja 2010; Marshall, 2000), en el año 2010 se aprueba la creación de una nueva entidad para el área metropolitana de Barcelona. La nueva entidad administrativa implica el intento de retorno del gobierno metropolitano después de la disolución de la anterior corporación, en 1987. El nuevo consorcio integrado por 36 municipios tiene una población de 3.225.058 habitantes y abarca una extensión de 633 km². Recogiendo las prioridades urbanísticas y de desarrollo económico de esta área, el Plan Metropolitano aprobado en el 2010, Visión 2020, identifica como áreas prioritarias de intervención para el horizonte 2020: la inversión en infraestructuras y la interconectividad; la promoción internacional y la atracción de capital inversor, de emprendedores y de turismo; la restructuración urbana a escala metropolitana para el desarrollo de actividades económicas vinculadas a la economía del conocimiento; y por último, el desarrollo de empresas y la expansión de las exportaciones (Pla Estratègic Metropolità de Barcelona, 2010).

Analizando las características de los proyectos existentes a escala metropolitana y el plan metropolitano se observa que el modelo de desarrollo planteado, aún en plena crisis económica, extrapola a escala metropolitana criterios de gobernanza y políticas que se han consolidado en la ciudad: los partenariados público-privados, la promoción de imagen, la provisión de infraestructura y la urbanización del suelo vinculada a la economía intensiva en conocimiento acompañada, siempre que es posible, de techo residencial de viviendas y oficinas. La promoción de sectores estratégicos vinculados a las nuevas tecnologías, la logística y el turismo se promocionan a través de la marca Barcelona (Informante 5, 2013).

Al inicio de la segunda década de siglo, bajo el concepto de El Triángulo Económico de Barcelona, una nueva marca promocional de la ciudad identifica tres clústeres de actividades económicas que reformulan proyectos existentes junto a otros nuevos, creando una nueva narrativa de marketing y creación de imagen a nivel metropolitano (Informante 6, 2012). Dos de estos clústeres se encuentran junto a los ríos que delimitan geográficamente la ciudad, los ríos Besòs i Llobregat. El tercer clúster está ubicado al oeste de la Región Metropolitana de Barcelona, en la comarca del Vallès.

El Area del Besòs es el clúster más cercano al centro histórico de Barcelona e incluye tres proyectos: el proyecto 22@Barcelona, ya descrito y ahora enmarcado en esta nueva estrategia promocional; un nuevo campus universitario y la reurbanización alrededor de una nueva estación de tren de alta velocidad. Siguiendo el modelo de los parques tecnológicos anglosajones e iniciado en el 2009, el campus proyecta nuevas instalaciones universitarias especializadas en ingeniería industrial y la atracción o generación de spin-off para activida- 
des empresariales. Sin embargo, fuertemente dependiente de la inversión pública, el proyecto se ve comprometido por las restricciones presupuestarias exigidas por la Unión Europea a España a partir del 2011. El segundo proyecto, la remodelación entorno a la nueva estación del tren para conectar Barcelona con las líneas de alta velocidad europeas, utiliza la restructuración de la nueva estación de tren de La Sagrera para actuar sobre 164 hectáreas. El proyecto implica la mejora substancial de este espacio gracias a la creación de una zona verde de 48 hectáreas, 20 hectáreas de nuevos equipamientos y una mejor conectividad entre dos barrios históricamente divididos por la línea férrea. A pesar de estos aspectos, mantiene un carácter fuertemente dependiente de las plusvalías del suelo, ya que el proyecto incluye 1,7 millones de metros cuadrados de techo, de los cuales $812.000 \mathrm{~m}^{2}$ están planificados como techo residencial, $380.000 \mathrm{~m}^{2}$ para oficinas y comercios y $55.500 \mathrm{~m}^{2}$ de techo hotelero -equivalentes a unas 800 habitaciones hoteleras. Esta dependencia dificulta su ejecución.

El segundo gran clúster metropolitano ubicado en el Área del Llobregat incluye proyectos vinculados a la mejora de la movilidad y el transporte, de los que destaca la ampliación del puerto y el aeropuerto de Barcelona. Dentro de esta área se identifican diferentes parques tecnológicos especializados en aeroespacial, agroalimentario y medioambiente. Los proyectos del puerto y el aeropuerto han sido históricamente difíciles de ejecutar por el carácter político de las grandes inversiones de infraestructura en España (Bel, 2011). Las limitaciones presupuestarias en infraestructura junto con los recortes presupuestarios en políticas de innovación y desarrollo resultantes de los criterios de austeridad, tanto del gobierno español como del catalán, han acrecentado las dificultades para estos proyectos.

Finalmente, el tercer clúster metropolitano se encuentra en la zona del Vallès, y reúne diversos parques tecnológicos vinculados a servicios y universidades. De carácter más económico, algunos proyectos también presentan criterios residenciales que difícilmente se podrán ejecutar en una coyuntura de crisis económica e inmobiliaria. El centro tecnológico más relevante es el Parc de l'Alba, centrado alrededor del Sincrotrón ALBA inaugurado en el año 2010. Este sincrotrón de última generación se planificó como uno de los proyectos estratégicos de Cataluña y tiene como objetivo convertirse en un potente motor de competitividad científica, tecnológica y empresarial en el sur de Europa. Supuso una inversión de más de 200 millones de Euros, y en el 2011, aún sin entrar en funcionamiento, comportó un coste de mantenimiento de 16 millones. Se calcula que en pleno rendimiento el coste será de entre un $20 \%$ o $30 \%$ más. Las nuevas restricciones presupuestarias, la dramática disminución de la inversión pública en I+D en España y la dependencia de la investigación española de los recursos públicos generan nuevos interrogantes sobre la viabilidad de este tercer clúster.

\section{REFLEXIONES FINALES: SOBRE LA NECESIDAD DE UN NUEVO PROCESO DE VIABILIDAD URBANA Y ECONÓMICA}

Desde principios de los años ochenta hasta la primera década del siglo veintiuno, el impacto de la política local de Barcelona ha sido extraordinario en cuanto a su capacidad de restructuración morfológica de la ciudad. El éxito en la proyección internacional de la ciudad es también indiscutible. Sin embargo, a pesar de poseer una imagen de política urbana con énfasis en aspectos sociales, la evolución urbanística de Barcelona no ha escapado al modelo identificado por Harvey, en el cual la gobernanza local, a través de la creciente importancia 
de los partearíamos público-privados, se ha transformado dando paulatinamente una mayor importancia a las políticas de desarrollo de carácter económico en detrimento de las políticas de carácter social. Esta tendencia se ha acentuado en el periodo de crisis, en la medida en que se ha recortado inversión en las ya muy mermadas políticas sociales.

A lo largo de varias décadas, el proceso de cooperación público-privado en Barcelona ha ido creando un reconocimiento de interdependencia y respeto mutuo entre el sector público y los sectores económicos dominantes. La existencia de unos agentes, con recursos tanto económicos como de gestión y conocimiento, ha favorecido la decisión de orientarse hacia unos objetivos concretos de crecimiento en los que la plusvalía del suelo ha sido el motor dominante, tanto para el sector privado con voluntad especulativa, como el sector público orientado hacia el crecimiento económico. Paulatinamente, el carácter dominante de estos objetivos en política urbana ha condicionado a su vez la identificación de nuevos agentes ricos en similares recursos. Como consecuencia de ello se ha ido generando una formación de preferencias en política local que ha primado los intereses de desarrollo económico especulativo de marcado carácter urbanístico, por encima de criterios de crecimiento económico redistributivo. Esta dinámica ha sido criticada por amplios colectivos, pero su capacidad de influir sobre las políticas urbanas ha sido limitada, ya que desde la década de los años noventa, la participación ciudadana en las políticas urbanas ha ido perdiendo capacidad de actuar e influir. Como demuestra el carácter de los proyectos ejecutados en la ciudad, más allá de los procesos participativos meramente protocolarios, amplios grupos de la ciudadanía sin recursos de capital o técnicos se han visto excluidos de los mecanismos generadores de visiones y estrategias de desarrollo.

La presente restructuración del capitalismo reabre pues la necesidad de replantearse la participación e inclusión de amplias capas de la ciudadanía en el proceso de gobernanza local, con el fin de identificar posibles alternativas de desarrollo económico y urbano, en un periodo de cambio que cuestiona la viabilidad de proyectos en curso. Tras el colapso de la «creación de espacio», en terminología de Harvey, se puede retornar a la necesaria «creación territorial». Los nuevos retos de futuro ligados a la necesidad de cambios de modelos productivos, a problemáticas medioambientales y energéticas, y a crecientes problemas de cohesión social requieren nuevos enfoques que amplíen la participación efectiva de la población (Casellas y Poli, 2013). La necesidad de ampliar la participación ciudadana en los procesos de gobernabilidad de la ciudad se hace pues necesaria para transferir el motor de desarrollo, desde el fallido proceso de urbanización del suelo a actividades económicas que capitalicen en iniciativas arraigadas en el territorio y generadas desde la sociedad civil.

El análisis histórico de la trasformación urbana en Barcelona permite observar una evolución paralela a la evolución del sistema capitalista, el cual ha entrado en crisis a partir del 2007. Se puede por ello argumentar que los modelos de gobernanza que han operado hasta la fecha, al margen de sus limitaciones en cuanto a justicia social, han dejado de ser efectivos incluso para los partenariados público-privados involucrados en el proceso. En un periodo de estancamiento económico o recesión en un número significativo de países, las políticas urbanas con dinámicas de colaboración público-privado crean nuevos interrogantes sobre su efectividad, ya que las condiciones macroeconómicas que los sustentaban se han agotado, pudiendo por ello representar mayores pérdidas de competitividad si se mantienen inalterados. 
Si el objetivo ya no puede ser crecer a toda costa, puede que debamos plantearnos la posibilidad de optimizar el uso de unos recursos locales que son escasos. Los nuevos retos implican la necesidad de un replanteamiento en el modelo de desarrollo y gobernanza de la ciudad, la cual debería desarrollar estrategias para incluir nuevos agentes y aportaciones generadas desde amplias capas de una sociedad civil plural.

\section{BIBLIOGRAFÍA}

ACEBILLO, J.A. (1999): «El modelo Barcelona desde el punto de vista urbanístico». En Pasqual Maragall (Ed.), Europa Próxima. Europa, Regiones y Ciudades Barcelona: Edicions Universitat de Barcelona. 223-248.

ALABART VILÀ, A. (2010): «Els Jocs Olímpics de Barcelona: oportunitats i riscos. Un balanç des de la sostenibilitat social». Biblio 3W. Revista Bibliográfica de Geografía y Ciencias Sociales, Universidad de Barcelona Vol. XV, $\mathrm{n}^{\circ} 895$ (4). <http://www.ub.es/ geocrit/b3w-895/b3w-895-4.htm>

ANDREU ACEBAL, M. (2011): «Poder, intereses económicos y modelo Barcelona». En Muntaner, J. Álvarez, F y Muxí, Z. Archivo crítico modelo Barcelona 1973-2004. Barcelona: Ajuntament de Barcelona. 53-57.

ANDREU ACEBAL, M. (2008): «Moviments socials i crítica al 'model Barcelona'. De l'esperança democràtica de 1979 al miratge olímpic de 1992 i la impostura cultural del 2004.» Diez años de cambios en el Mundo, en la Geografía y en las Ciencias Sociales, 1999-2008. Actas del X Coloquio Internacional de Geocrítica, Universidad de Barcelona. http://www.ub.es/geocrit/-xcol/225.htm

ASOCIACIÓN DE PROMOTORES DE BARCELONA- APCE (2013): «La crisis se ceba en Diagonal Mar». Blog. http://apcebcn.cat/content/index.php?option=com_content\&tas $\mathrm{k}=$ view\&id=9110\&Itemid=9. [Consultado, 31 Noviembre 2013].

BALCELLS, S. (1986): Com Desfer Catalunya: Barcelona, 92. Sant Boi de Llobregat: Lluita.

BLANCO, I. (2009): «Does a 'Barcelona Model' Really Exist? Periods, Territories and Actors in the Process of Urban Transformation». Local Government Studies, 35 (3), 355-369.

BEL, G. (2011): «Infrastructure and nation building: The regulation and financing of network transportation infrastructure in Spain». Business History, 53 (5), 688-705.

BOHIGAS, O. (1985): Reconstrucció de Barcelona. Barcelona: Edicions 62.

BOHIGAS, O. (1999):«Valorización de la periferia y recuperación del centro». En Pasqual Maragall (Ed.), Europa Próxima. Europa, Regiones y Ciudades Barcelona: Edicions Universitat de Barcelona. 199-214.

BOHIGAS, O. (2004): Contra la Incontinència Urbana. Reconsideració Moral de l'Arquitectura i la Ciutat. Barcelona: Institut d’Edicions de la Diputació de Barcelona.

BORJA, J. y otros (1996): Barcelona, un modelo de transformación urbana (1980-95). Ayuntamiento de Barcelona: UNDP-World Bank.

BORJA, J. (2005): «Un Futuro urbano con un corazón antiguo». Geograficando, 1 (1), 11-22.

BORJA, J. (2007): «Revolución y contrarrevolución en la ciudad global: las expectativas frustradas por la globalización de nuestras ciudades». EURE. Revista Latinoamericana de Estudios Urbano Regionales. 33 (100), 35-50. 
BORJA, J. (2010): Llums i Ombres de l’Urbanisme a Barcelona. Barcelona: Biblioteca Universal Empúries.

BURRIEL, E. (2008): «La «década prodigiosa» del urbanismo español (1997-2006)». Scripta Nova. Revista electrónica de Geografía y Ciencias Sociales, 2008, vol. XII, n ${ }^{\circ}$ 270 (64). <http://www.ub.edu/geocrit/sn/sn-270/sn-270-64.htm>.

BURRIEL, E. (2011): «Subversion of land-use plans and the housing bubble in Spain». Urban Research \& Practice, vol. 4, n.3, p. 232-249.

BUSQUETS, J. (2004): Barcelona. La Construcción Urbanística de la Ciudad Compacta. Barcelona: Ediciones del Serbal.

CALAVITA N. y FERRER, A. (2000): «Behind Barcelona's success story. Citizen movements and planner's power». Journal of Urban History, 6, 793-807.

CAPEL, H. (2005): El Modelo Barcelona: Un Examen Crítico. Barcelona: Ediciones del Serbal.

CAPEL, H. (2007): «El debate sobre la construcción de la ciudad y el llamado «Modelo Barcelona». Scripta Nova. Revista Electrónica de Geografía y Ciencias Sociales. Barcelona: Universidad de Barcelona, 15 de febrero de 2007, vol. XI, núm. $233<$ http://www.ub.es/ geocrit/sn/sn-233.htm>.

CARAVACA, I.; GONZÁLEZ, G. (2009): «Las redes de colaboración como base del desarrollo territorial». Scripta Nova. Revista Electrónica de Geografía y Ciencias sociales. Barcelona: Universidad de Barcelona, 1 de mayo de 2009, vol. XIII, núm. 289<http:// www.ub.es/geocrit/sn/sn-289.htm>.

CASELlAS, A (2003): «The Barcelona Model? Agents, Policies and Planning Dynamics in Tourism Development». Tesis Doctoral. Department of Urban Planning and Policy Development. Rutgers, the State University of New Jersey.

CASELLAS, A (2005): «Aproximaciones teóricas para el análisis de la transformación urbana: Urban Regime Analysis en el contexto europeo». Documents d'Anàlisi Geogràfica, 46, 139-147.

CASELLAS, A (2006): «Las limitaciones del «modelo Barcelona». Una lectura desde Urban Regime Analysis». Documents d'Anàlisi Geografica, 48, 61-81.

CASELLAS, A. (2007): «Gobernabilidad, participación ciudadana y desarrollo económico: adaptaciones locales a estrategias globales». Scripta Nova. Revista Electrónica de Geografia y Ciencias Sociales, Barcelona: Universidad de Barcelona, 10 de julio de 2007, vol. XI, núm. $243<$ http://www.ub.es/geocrit/sn/sn-243.htm>. [ISSN: 1138-9788].

CASELLAS, A. y PALLARES-BARBERA, M. (2009): «Public-sector intervention in embodying the new economy in inner urban areas: the Barcelona experience». Urban Studies, 46 (5\&6), 1137-1155.

CASELLAS, A. y POLI, C. (2013): «Planificación urbana e innovación: De la tecnología y la práctica profesional a la innovación social y política». Documents d'Anàlisi Geogràfica, 59, 3, 435-454.

CRUZ GALLACH, H. y MARTÍ-COSTA M. (2010): «Conflictos urbanísticos y movilizaciones ciudadanas: reflexiones desde Barcelona». Finisterra, 90, 111-132.

DELGADO, M. (Ed.). (2004): La otra cara del Fòrum de les cultures S. A. Barcelona: Edicions Bellaterra.

DELGADO, M. (2005): Elogi del Vianant. Del «Model Barcelona» a la Barcelona Real. Barcelona: Edicions de 1984. 
DELGADO, M. (2007): La ciudad mentirosa. Fraude y miseria del «modelo Barcelona». Madrid: Los libros de la Catarata.

DOT, E., CASELLAS, A. y PALLARES-BARBERA, M. (2010): «L'ambigüitat de la producció intensiva en coneixement: el nou espai econòmic del poblenou». Documents d'Anàlisi Geogràfica, 56 (3), 389-408.

EL CONFIDENCIAL (2013): «El 'banco malo' irlandés busca comprador a un gran centro comercial de Barcelona». 6 Agosto 2013. http://www.elconfidencial.com/ empresas/2013-08-06/el-banco-malo-irlandes-busca-comprador-a-un-gran-centro-comercial-de-barcelona_15679/ [Consultado: 25 Octubre 2013].

EL TRIANGULO ECONOMICO DE BARCELONA/ CATALONIA (2013): (The Mediterranean Innovation Hub) (http://www.barcelonaeconomictriangle.cat/) [Consultado: 22 Octubre 2013].

ESTEBAN, J (1999): «El projecte urbanístic. Valorar la perifèria i recuperar el centre. Model Barcelona». Quaderns de Gestió. Barcelona: Aula Barcelona.

ESTEBAN, J (2003): La ordenación urbanística: conceptos, herramientas y prácticas. Barcelona: Diputació de Barcelona-Electa.

FARINÓS, J. (2008): «Gobernanza territorial para el desarrollo sostenible: estado de la cuestión y agenda». Boletín de la Asociación de Geógrafos Españoles, 46, págs. 11-32.

FLORIDA, R. (2004): Cities and the Creative Class. New York: Routledge.

FLORIDA, R., GULDEN, T., and MELLONDER, C. (2007): «The Rise of the Mega Region». Toronto: Joseph L. Rotman School of Management, University of Toronto.<http://www. rotman.utoronto.ca/userfiles/prosperity/File/Rise.of.\%20the.Mega-Regions.w.cover.pdf>

GARCÍA, A. (2013): «El branding de ciudad: la promoción del modelo Barcelona y su proyección como marca». Tesis Doctoral. Universitat Politècnica de Catalunya. Barcelona, marzo 2013.

GEROMETTA, J.; HÄUSSERMANN, H.; LONGO, G. (2005): «Social innovation in urban governance: strategies for an inclusive city». Urban Studies, 42, 2007-2021.

GILBERT, A. y GUGLER, J. (1992): Cities, Poverty and Development. Oxford: Oxford University Press.

GONZÁLEZ, S. (2011): «Bilbao and Barcelona in motion. How urban regeneration models travel and mutate in the global flows of policy tourism». Urban Studies, 48 (7), $1397-$ 1418 ,

GRANGE, J. (1999): The City: an Urban Cosmology. Albany: State University of New York Press.

GRUP DE PATRIMONI INDUSTRIAL DEL FÒRUM RIBERA BESÒS (2005a): «Un patrimoni únic, un futur brillant, un model de fer ciutat. Can Ricart-Parc Central de Barcelona. Nou projecte». Biblio 3W. Revista Bibliográfica de Geografía y Ciencias Sociales, Universidad de Barcelona, 30 de abril 2005a, vol. X, n 580. <http://www.ub.es/geocrit/ b3w-580.htm>

GRUP DE PATRIMONI INDUSTRIAL DEL FÒRUM RIBERA BESÒS (2005b): «Proposta de Pla Integral de Patrimoni Industrial de Barcelona». Biblio 3W. Revista Bibliográfica de Geografía y Ciencias Sociales, Universidad de Barcelona, 5 de mayo de 2005b, vol. X, no $581<$ http://www.ub.es/geocrit/b3w-581.htm> 
HACKER, J. (2006): The Great Risk Shift: The Assault on American Jobs, Families, Health Care, and Retirement and How You Can Fight Back. Oxford: Oxford University Press.

HAJER, M. (2003): «Policy without polity? Policy analysis and the institutional void». Policy Sciences. 36, 175-195.

HALL, P. (1998): Cities in Civilisation: Culture, Innovation and Urban Order. London: Weidenfeld.

HARVEY, D. (2011): «Crisis, Geographic Disruptions and the Uneven Development of Political Responses». Economic Geography. 87, (1), 1-22.

HARVEY, D. (2010): The Enigma of Capital and the Crises of Capitalism. Oxford: Oxford University Press, USA, 2 edition.

HARVEY, D. (1989): «From Managerialism to entrepreneurialism: the transformation in urban governance in late capitalism». Geografiska Annaler, 71 B (1), 3-17.

HEALEY, P. (2007): Urban Complexity and Spatial Planning: Towards a relational planning for our times. London: Routledge.

HOOGHE, L. y MARKS, G. (2003): «Unravelling the Central State, But How?» American Political Science Review, 97 (2), 233-43.

JESSOP, B. (2002): «Liberalism, neoliberalism and urban governance: A state-theoretical perspective». Antipode, 34, 452-472.

LE GALÈS, P. (2002): European Cities: Social Conflicts and Urban Governance. Oxford: Oxford University Press.

MARAGALL, P. (1999): «El evento como estrategia del desarrollo urbano: Los Juegos Olímpicos del '92». En Pasqual Maragall (Ed.), Europa Próxima. Europa, Regiones y Ciudades. Barcelona: Edicions Universitat de Barcelona. 249-254.

MARAGALL, P. (2008): Oda Inacabada. Barcelona: RBA.

MARSHALL, T. (2000): «Urban planning and governance: is there a Barcelona model?» International Planning Studies, 5 (3), 299-319.

MARSHALL, T. (Ed). (2004): Transforming Barcelona. London, Routlegge.

MARTÍ-COSTA, M. y PRADEL i MIQUEL, M. (2011): «The knowledge city against urban creativity? Artists' workshops and urban regeneration in Barcelona». European Urban and Regional Studies, 18 (4), 1-17.

MAURI, L. y URÍA, L. (1998): La Gota Malaia. Barcelona: Edicions 62.

McNEILL, D. (1999): Urban Change and the European Left. Tales from the New Barcelona. New York: Routledge.

McNEILL, D. (2003): «Mapping the European Left: the Barcelona Model». Antipode. 35 (1), 74-94.

MÉNDEZ, R. (2013): Estrategias de innovación para el desarrollo y la resiliencia de ciudades medias». Documents d'Anàlisi Geogràfica, 59 (3), 481-499.

MILIO, S. (2013): T»he conflicting effects of multi-level governance and the partnership principle: Evidence from the Italian experience». European Urban and Regional Studies. published online 13 August 2013 DOI: 10.1177/0969776413493631

MOIX, Ll. (1994): La Ciudad de los Arquitectos. Barcelona: Crónicas Anagrama.

MONCLÚS, F.J. (2003): «The Barcelona Model: an original formula? From «Reconstruction» to Strategic Urban Projects (1979-2004)». Planning Perspectives, 18 (4), 399-421.

MONTANER, J. M. (2003): Repensar Barcelona. Barcelona: Edicions UPC. 
MONTANER, J. M. (2004): «Argumentos de la Barcelona poliédrica». En Costa, Guim. Barcelona 1992-2004, Barcelona: Gustavo Gili, 19-23.

MOULEART, F.; MARTINELLI, F.; GONZÁLEZ, S.; SWYNGEDOUW, E. (2007): «Introduction: social innovation and governance in European cities». European Urban and Regional Studies, 14 (3), 195-208.

PAREJA-EASTAWAY, M. y PIQUÉ, J.M. (2011): «Urban regeneration and the creative knowledge economy: the case of 22@Barcelina» Journal of Urban Regeneration and Renewal, 4 (4), 319-237.

PÉREZ, C. (2006): «Un grupo inversor irlandés compra Diagonal Mar por 300 millones de euros». El País. 25 Mayo 2006. http://elpais.com/diario/2006/05/25/catalunya/1148519240_850215.html. [Consultado: 3 Septiembre 2013].

PIERRE, J. y PETERS, B. G. (2000): Governance, Politics and the State. Basingstoke: Macmillan.

PLA ESTRATEGIC METROPOLITA DE BARCELONA (2010): http://www.pemb.cat/ visio-2020/ [Consultado: 3 Octubre 2013].

RAVENTOS, F. (1998): «Més d'una dècada de planificació estratègica de ciutats». En. 10 Anys de Planificació Estratègica a Barcelona (1988-1998) Barcelona: Associació Pla Estratègic Barcelona 2000. 17-28.

RAVENTOS, F. (2000): La Col.laboració Publicoprivada. Barcelona: Aula Barcelona.

SABATÉ BEL, J. y TIRONI RODÓ, M (2008): «Globalización y estrategias urbanísticas: un balance del desarrollo reciente de Barcelona». Cuaderno Urbano, Espacio, Cultura, Sociedad, 7(7), 233-260.

SANTACANA, F. (1999): «La planificació estratègica urbana: 1'experiència de Barcelona». En Pasqual Maragall (Ed.), Europa Próxima. Europa, Regiones y Ciudades. Barcelona: Edicions Universitat de Barcelona. 261-266.

SASSEN, S. (1991): The Global City: New York, London and Tokyo. Princeton: Princeton University Press.

SATTERTHWAITE, D. (2009): «The implications of population growth and urbanization for climate change». Environment and Urbanization, 21 (2), 545-567.

SWYNGEDOUW, E. (2005): «Governance innovation and the citizen: The Janus face of governance-beyond-the-state». Urban Studies, 42 (11), 1991-2006.

TAPADA-BERTELI, T. \& ARBACI, S. (2011): «Proyectos de regeneración urbana en Barcelona contra la segregación socio-espacial (1986-2009): ¿solución o mito?» ACE: Architecture, City and Environment, 6 (17), 187-222.

UNIÓ TEMPORAL DE ESCRIBES (Eds). (2004): Barcelona, Marca Registrada. Un model per desarmar. Barcelona: PEPA Virus.

SCOTT, A. J. (2008): Social Economy of the Metropolis: Cognitive-Cultural Capitalism and the Global Resurgence of Cities. Oxford: Oxford University Press.

SMITH, N. (1987): «Gentrification and the rent-gap». Annals of the Association of American Geographers. 77 (3), 462-465.

STOKER, G. (1998): «Governance as Theory». International Social Science Journal, 17-28.

$22 @ B A R C E L O N A . P r e s e n t a c i o ́ n . h t t p: / / w w w .22 b a r c e l o n a . c o m / c o n t e n t / b l o g c a t e-$ gory/49/280/lang,es/ [25 Enero 2012] 
